\title{
Erratum to: Relations Among Self-concealment, Mindfulness, and Internalizing Problems
}

\author{
Joshua Edmonds • Akihiko Masuda • Erin C. Tully
}

Published online: 26 June 2013

(C) Springer Science+Business Media New York 2013

\section{Erratum to: Mindfulness \\ DOI 10.1007/s12671-013-0204-z}

In the original version of the article, there are three errors. First, the description of the association between selfconcealment and internalizing problems in Abstract should be "High levels of self-concealment predicted higher levels of depression and anxiety above the effects of mindfulness, age, and ethnicity for both men and women, and high levels of self-concealment predicted higher levels of somatization for women". Second, the description of associations among mindfulness, self-concealment, and internalizing problems in the second paragraph on page 5 (i.e., third paragraph in
Result) should be, "These models support the hypothesis that lower levels of mindfulness and higher levels of selfconcealment predict significantly higher levels of depression and anxiety for both sexes and higher levels of somatization for women above the effects of each other and the covariates. Finally, the description of the association between selfconcealment and internalizing problems in the first paragraph on page 6 (i.e., first paragraph in Discussion) should be, "Also as expected, high levels of self-concealment predicted high levels of depression and anxiety above the effects of mindfulness, age, and ethnicity for both men and women, and high levels of self-concealment predicted higher levels of somatization for women".

The online version of the original article can be found at http://dx.doi.org/ 10.1007/s12671-013-0204-z. 